\title{
Decodificaç̃o com Busca Restrita de Sistemas CPM Combinados com Códigos Convolucionais em Canais com Desvanecimento
}

\author{
Cecílio J. L. Pimentel \\ Departamento de Informática \\ Universidade Federal de Pernambuco \\ Caixa Postal 7851 - Recife - PE, 50732-970, Brasil \\ Weiler A. Finamore \\ CETUC \\ PUC-RIO \\ Rua Marquês de São Vicente, 255 \\ 22453 - Rio de Janeiro - RJ
}

\begin{abstract}
Este trabalho apresenta o desenvolvimento de um modelo em tempo discreto, para a análise através de simulação em computador digital do desenpenho de um sistema de comunicações que utiliza modulação CPM (Continuous Phase Modulation j combinada com código convolucional (CC/CPM) em presença de desvanecimento. A seleção do esquema CC/CPM ótimo foi realizada utilizando um algoritmo eficiente para o cálculo da distância euclidiana quadrática mínima. Novos códigos com o codificador convolucional com estrutura realimentada são apresentados. Em alguns casos, estes apresentam valores de distância euclidiana mínima melhores em relação aos códigos CC/CPM previamente publicados, que usam o codificador convolucional com estrutura feedforward. Geralmente os esquemas CC/CPM eficientes são caracterizados por uma codificação com um número elevado de estados o que torna a decodificação por máxima verossimilhança impraticável, mesmo usando um algorítmo eficiente como o de Viterbi. Neste trabalho o decodificador utiliza um algoritmo subótimo de busca restrita, o Algoritmo-M. São realizadas simulações com o Algoritmo-M e compara-se com o Algoritmo de Viterbi. Verifica-se que o AlgoritmoMé bastante mais eficiente que o Algoritmo de Viterbi, em várias situações de interesse prático.
\end{abstract}

\section{Introdução}

Implantar um sistema de comunicaçōes móveis via satélite corresponde a estabelecer comunicações em um ambiente bastante hostil quando comparado com comunicações fixas. Estes sistemas são limitados em faixa e em potência e os sinais recebidos estão sujeitos a, além de outras perturbações, desvanecimento. $O$ desvanecimento corresponde a flutuações de natureza aleatória na amplitude do sinal recebido. Estas flutuações são provocadas principalmente por múltiplos percursos tomados pelo sinal transmitido antes de chegar ao receptor; por atenuações introduzidas por obstáculos (vegetações, por exem- 
plo) e também em virtude do movimento da viatura. Acrescente-se a estas dificuldades, a necessidade de comunicaçōes com taxas elevadas e com alta confiabilidade.

Diversos aspectos devem ser considerados para o estabelecimento de um sistema dessa natureza, como por exemplo, uso de códigos corretores de erro. Técnicas que tratam a codificação e modulação de forma integrada têm se demonstrado eficientes em termos de ocupação de faixa e utilização de potência [1]. Em particular a técnica denominada CPM é contemplada como um procedimento eficiente para ser usado em presença de desvanecimento, sendo portanto de interesse a análise do seu desempenho.

É interessante ressaltar que o esquema CPM não realiza apenas a modulação como pode parecer inicialmente. De fato o processo de codificação é realizado simultaneamente com o processo de modulação, quando se impõe a continuidade de fase do sinal modulado. O conceito moderno de codificação envolve a imposição de padrões no sinal transmitido. Dessa forma o receptor que conhece todos os possíveis padrões que podem ser transmitidos, escolhe aquele que é mais próximo do sinal recebido. Este procedimento engloba o codificador e o modulador em uma única função, como também, o demodulador e o decodificador.

No sistema CPM a informação é enviada na fase do sinal passa-faixa transmitido pelo modulador. O sinal modulado possui envoltória constante e a fase é uma função contínua no tempo. A fase do sinal transmitido, em cada intervalo de sinalização, depende da fase em intervalos anteriores. Esta correlação entre as fases em intervalos sucessivos introduz uma memória no processo de modulação (que dará origem a definição do estado do modulador), devendo esta ser explorada para conseguir esquemas eficientes em termos de consumo de potência e ocupação de faixa.

Diversos trabalhos têm investigado a precodificação dos símbolos de entrada do modulador CPM. Uma das formas eficientes de aumentar a memória da fase do sinal modulado é através da combinação da modulação CPM e codificação convolucional (CC/CPM). Esta combinação pode ser interpretada como um código concatenado, visto que a modulação CPM também é um código de treliça. Em [2, 3] o codificador convolucional com estrutura feedforward foi utilizado para a busca do código ótimo. Mostrou-se que esta combinação conduz a um significante aumento conjunto potência-largura de faixa. Os resultados obtidos, quando o codificador convolucional com estrutura realimentada é combinado com o modulador CPM é apresentado neste trabalho.

A combinação CC/CPM conduz a sistemas complexos e, no processo de decodificação a busca exaustiva da seqüência transmitida pode tornar-se um problema computacionalmente impraticável.

Neste trabalho examina-se o desempenho de sistemas CC/CPM em presença de desvanecimento. $\mathrm{O}$ decodificador utiliza um algoritmo subótimo para a busca da sequiência transmitida. Este algoritmo é denominado Algoritmo-M. A técnica utilizada para a análise de desempenho é a simulação em computador digital. 
A técnica de simulação requer que os diversos elementos do sistema sejam modelados de forma adequada para a implementação em computador digital. Uma maneira de realizar esta adequação é usar para as diversas formas de onda presentes no sistema a representação em banda básica, através de suas amostras em instantes de tempo discretos, igualmente espaçadas.

Este trabalho está organizado da seguinte forma. Na Seção 2 apresenta-se uma descrição detalhada do esquema Codificador Convolucional/Modulador CPM combinados. O processo estocástico que modela o efeito do desvanecimento é, nesta seção, caracterizado do ponto de vista estatístico e um modelo matemático é estabelecido para este processo. É descrito um esquema que produz, em tempo discreto, uma sequiência de variáveis aleatórias (va) complexas que modela o desvanecimento. A validade do modelo é comprovada a partir de comparação com medidas experimentais.

A Seção 3 é dedicada ao cálculo da distância euclidiana mínima para o código convolucional com uma estrutura realimentada combinado com modulação CPM. Os resultados apresentados foram obtidos com a implementação do algoritmo de busca da distância euclidiana mínima para códigos em treliça publicado em [4].

A Seção 4 apresenta os resultados de simulação de um sistema de comunicações que emprega o CC/CPM em presença de desvanecimento. Observa-se o desempenho do sistema CC/CPM quando o Algoritmo-M é usado na decodificação e compara-se com resultados semelhantemente obtidos com o uso do algoritmo de decodificação ótimo (Algoritmo de Viterbi).

\section{Caracterização do Sistema de Comunicação}

A Fig.1 apresenta o modelo equivalente em banda básica de um sistema de comunicações que será analisado neste trabalho.

A fonte de informação produz uma sequiência de símbolos binários independentes e identicamente distribuídos, $\underline{b}=\left\{\ldots, b_{k-1}, b_{k}, b_{k+1, \ldots}\right\}$, a uma taxa de informação $1 / T_{b}$ que seráentr adadeumconver sorsér ie-paralelo.

Durante o $(k+1)$-ésimo intervalo de sinalização $\tau_{k}=\{t: k T \leq t<(k+1) T\}$ a saída do conversor é caracterizada pelo vetor $\underline{c}_{k}=\left(c_{k}^{1, \cdots, \cdot} c_{k}^{l}\right)$, composto de $l$ bits de informação $\left(\mathrm{T}=l \mathrm{~T}_{\mathrm{b}}\right.$ e $1 / \mathrm{T}$ é a taxa de sinalização). Cada componente do vetor $\underline{\mathrm{c}}_{\mathrm{k}}$, $\mathrm{k}$ inteiro é da forma

$$
c_{k}^{i}=b_{k} l+i-1 \quad ; i=1, \ldots, l
$$


$O$ vetor $\underline{c}_{k}$ é a entrada de um codificador convolucional de taxa $R_{c}=\mathscr{V} / \mathrm{n}$ bits de informação / bits codificados, produzindo na sua saída o vetor de $\mathrm{n}$ bits $\underline{v}_{\mathrm{k}}=\left(v_{\mathrm{k}}^{1, \cdots} v_{\mathrm{k}}^{\mathrm{n}}\right)$. Neste trabalho considera-se o codificador convolucional com uma estrutura realimentada. O diagrama genérico de um codificador com estrutura realimentada de taxa $R_{c}=m /(m+1)$ e memória de tamanho $v$ é mostrado na Fig.2. Para este codificador, os polinômios geradores são da forma [1] ou

$$
\begin{array}{ll}
\mathrm{H}^{\mathrm{j}}(\mathrm{D})=0 & \widetilde{\mathrm{m}}<\mathrm{j} \leq \mathrm{m} \\
\mathrm{H}^{\mathrm{j}}(\mathrm{D})=0+\mathrm{h}_{-1}^{\mathrm{j}} \mathrm{D}^{v-1}+\ldots+\mathrm{h} \cdot \mathrm{D}+0 & 1 \leq \mathrm{j} \leq \widetilde{\mathrm{m}} \\
\mathrm{H}^{0}(\mathrm{D})=\mathrm{D}^{\nu}+\mathrm{h}_{v^{-1}-1}^{0} \mathrm{D}^{v-1}+\ldots+\mathrm{h}_{[}^{0} \mathrm{D}+1 &
\end{array}
$$

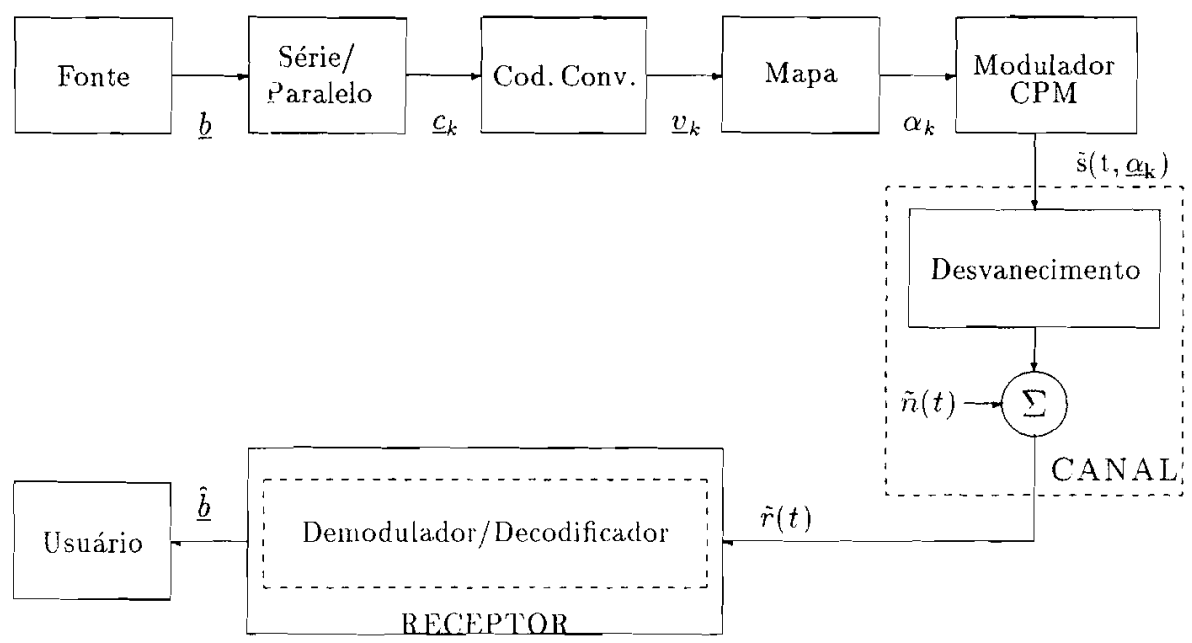

Figura 1 - Diagrama representando em banda básica um sistema de comunicação digital

O estado do codificador no intervalo $\tau_{\mathrm{k}}$ é caracterizado pelo vetor $\Omega_{\mathrm{k}}=\left(\Omega_{\mathrm{k}}^{1}, \ldots, \Omega_{\mathrm{k}}^{v}\right)$, contendo $v$ componentes. Cada componente $\Omega_{\mathrm{k}}^{\mathrm{i}}$ representa o conteúdo das células denotadas por $\mathcal{I}$ na Fig.2, obtidas a partir de uma combinação linear dos símbolos de informação $c_{j}^{i}$ (paraj $<$ k e $1 \leq i \leq \tilde{m})$.

O vetor $\underline{v}_{\mathrm{k}}$ é mapeado em um símbolo $\alpha_{\mathrm{k}}$ pertencente ao alfabeto Q-ário dado pelo conjunto $\{ \pm 1, \pm 3, \ldots \pm \mathrm{Q}-1\}$, onde $\mathrm{Q}=2^{\mathrm{V}}$. O símbolo $\alpha_{\mathrm{k}}$ é o símbolo de entrada do modulador CPM. Neste trabalho é utilizado mapeamento denominado natural definido por 


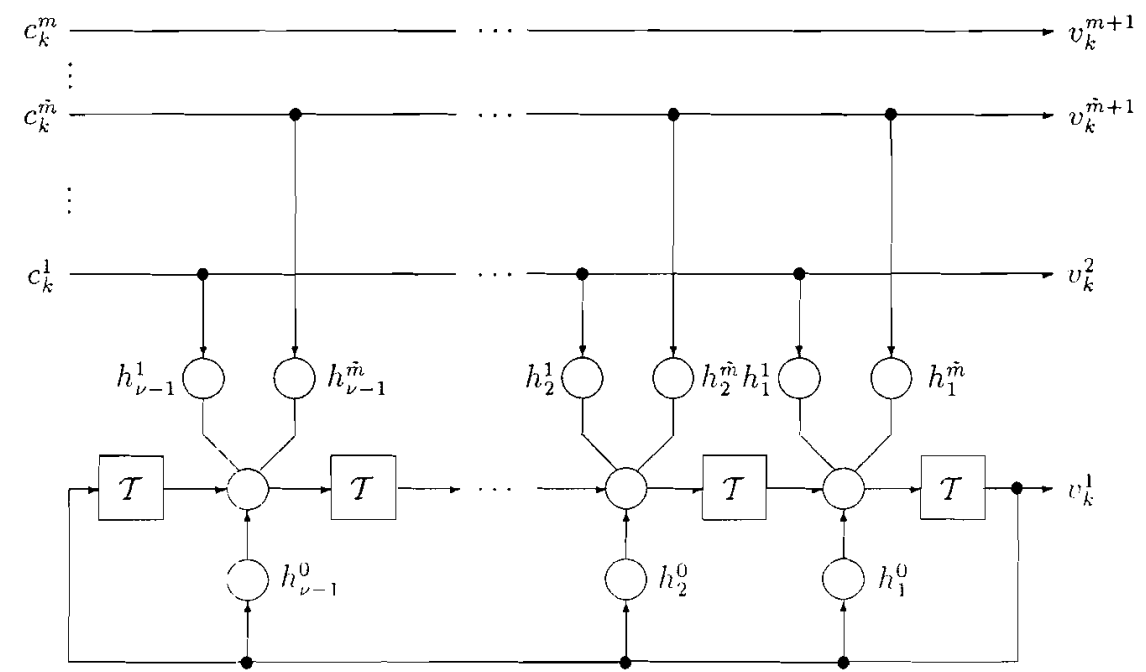

Figura 2 - Estrutura do Codificador Convolucional Sistemático, com estrutura realimentada.

$$
\alpha_{k}=\left\{\sum_{i=1}^{n} v \mathrm{i}^{\mathrm{l}} 2^{\mathrm{l}+1-\mathrm{i}}\right\}-\mathrm{Q}+1
$$

O simbolo $\alpha_{k}$ juntamente com o estado do modulador produzem a forma de onda $\widetilde{s}\left(\mathrm{t}, \underline{\alpha}_{\mathrm{k}}\right)$ correspondente à envoltória complexa do sinal CPM que será transmitido através do canal no intervalo $\tau_{\mathrm{k}}$. As perturbações introduzidas pelo canal no sinal transmitido são representadas pelo processo estocástico a(t), que caracteriza o processo de desvanecimento multiplicativo e por $\tilde{\mathrm{n}}(\mathrm{t})$, caracterizando a envoltória complexa de um ruído Gaussiano branco, de média nula e densidade espectral de potência unilateral plana com valor $\mathrm{N}_{0}$.

O efeito da transmissão de um sinal através de um canal com desvanecimento pode ser entendido como multiplicar por um fator a(t) a envoltória complexa do sinal transmitido $\widetilde{s}(t, \underline{\alpha})\left[\underline{\alpha}\right.$ é a sequência de sîmbolos $\left.\left(\ldots, \alpha_{k}, \alpha_{k+1, \ldots}\right)\right]$. A envoltória complexa do sinal recebiḍo $\widetilde{r}(\mathrm{t})$ é expressa simplesmente por $\widetilde{\mathrm{r}}(\mathrm{t})=\mathrm{a}(\mathrm{t}) \widetilde{\mathrm{s}}(\mathrm{t}, \underline{\alpha})+\widetilde{\mathrm{n}}(\mathrm{t})$.

Na recepção, o decodificador de máxima verossimilhança determina que a seqüência supostamente transmitida, é aquela que maximiza a métrica $\lambda(\underline{\alpha})$ (logaritmo da função de verossimilhança). Esta função, corresponde a densidade de probabilidade do sinal observado $\widetilde{r}(t)$, condicionado a sequiência $\underline{\alpha}$. Quando a única perturbação presente no sistema é o ruído aditivo Gaussiano branco (canal AWGN), a métrica tem a seguinte expressão [5]:

$$
\lambda(\underline{\alpha})=-\frac{2}{\mathrm{~N}_{0}} \int_{-\infty}^{\infty}[\widetilde{\mathrm{r}}(\mathrm{t})-\widetilde{\mathrm{s}}(\mathrm{t}, \underline{\alpha})]^{2} \mathrm{dt}
$$

Revista da Sociedade Brasileira de Telecomunicações 
A métrica acima, conduz a um receptor que decide sobre a possível sequiência transmiti'a baseado no critério da distância euclidiana quadrática mínima. Para sistemas, cujos silais transmitidos têm a mesma energia, este critério corresponde ao critério de máxima :orrelação [5]. No caso de sistemas em presença de desvanecimento, a determinação da nétrica ótima é um problema de difícil solução. Neste trabalho, adotou-se como métrica subótima), a distância euclidiana quadrática mínima, definida em (4).

J decodificador, a partir da observação do sinal $\widetilde{\mathrm{r}}(\mathrm{t})$ recebido, realiza uma decisão que :onduz a uma seqüência $\underline{\underline{b}}$ supostamente transmitida. Neste trabalho, o algoritmo de de:odificação utilizado, Algoritmo-M, é subótimo e não realiza a busca da sequiência transnitida de forma exaustiva conforme feito com o Algoritmo de Viterbi.

\subsection{Caracterização do Desvanecimento}

Vesta seção as atenuações variantes no tempo, sofridas pelo sinal recebido por uma viaura em um sistema de comunicações móveis via satélite são caracterizados do ponto de vista estatístico.

O sinal recebido por uma viatura pode ser modelado como sendo constituído por uma parcela correspondente a uma componente de visada direta, atenuada por obstáculos, e uma parcela correspondente à componente multi-percurso originada pela combinação de sinais refletidos por obstáculos e pelo terreno.

Em um dado instante, o fator a(t) que caracteriza o desvanecimento é modelado como uma variável aleatória (va) complexa, a $\mathrm{e}^{\mathrm{j} \theta}$, dada pela soma de duas variáveis aleatórias complexas independentes.

$$
a e^{j \theta}=z e^{j \Phi_{0}}+w e^{j \Phi}
$$

Em (5), $\phi_{0}$ e $\phi$ são va's uniformes no intervalo $[0,2 \pi]$. A va z corresponde ao efeito da atenuação introduzida pela vegetação e outros tipos de obstrução sobre a componente de visada direta do sinal recebido e é modelada como uma va com função densidade de probabilidade (fdp) Log-Normal. Esta va zé da forma

$$
\mathrm{z}=\mathrm{e}^{\mathrm{u}}
$$

onde $\mathrm{u}$ é uma va Gaussiana com média $\mu_{0} \mathrm{e}$ variância $\mathrm{d}_{0}$. A variável aleatória w corresponde ao efeito de multi-percurso é modelada como uma va com fdp de Rayleigh de média $\sqrt{\pi \mathrm{b}_{0} / 2}$ e variância $(2-\pi / 2) \mathrm{b}_{0}$.

As va's definidas em dois instantes de tempo distintos no processo a(t) são correlacionadas. Este modelamento do fator de desvanecimento foi proposto por Loo [6] e constitui

70 
um modelo adequado para representar o fenômeno do desvanecimento em comunicações móveis via satélite, conforme demonstrado através da comparação dos resultados obtidos com o modelo teórico com os resultados obtidos experimentalmente [7].

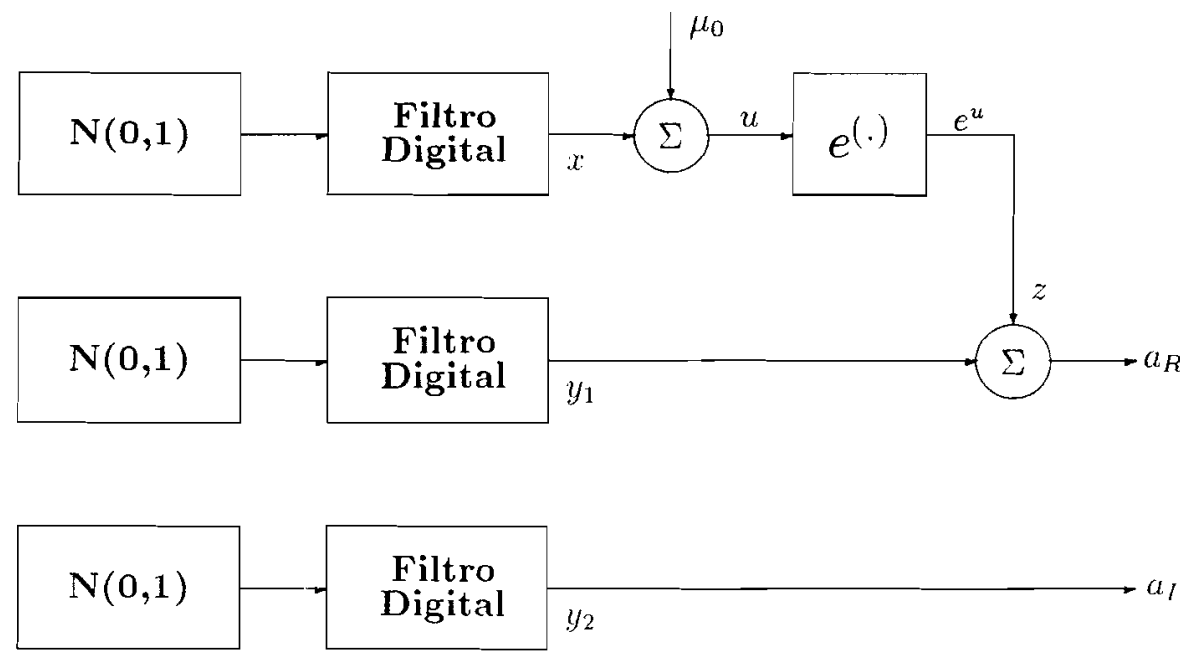

Figura 3 - Modelo de Simulador de Desvanecimento

O modelo estatístico apresentado é útil para o desenvolvimento de simuladores que reproduzem os efeitos de propagação em comunicações móveis e permitem a simulação desses sistemas em computador digital. Uma estrutura, sugerida em [8], capaz de incorporar os efeitos do desvanecimento no canal à simulação em computador digital é mostrada na Fig.3. As partes real e imaginária do fator de desvanecimento definido em (5) são produzidas, em tempo discreto, através das variáveis $\mathrm{a}_{\mathrm{R}} \mathrm{e} \mathrm{a}_{\mathrm{I}}$ indicadas na figura. Os blocos caracterizados por $\mathrm{N}(0,1)$ correspondem a geradores de variáveis aleatórias Gaussianas independentes. de média nula e variância unitária. As sequiências x. $y_{1}, y_{2}$ são va's Gaussianas de média zero e as variâncias são respectivamente $\mathrm{d}_{0}, \mathrm{~b}_{0}, \mathrm{~b}_{0}$. A sequiência de variáveis $Z_{\mid}^{\backslash}$ corresponde a uma seqüência de va's Log-Normais correlacionadas.

Os filtros digitais controlam a largura de faixa do desvanecimento e são responsáveis pela introdução de correlação entre as amostras da seqüência de saída do simulador. Neste trabalho foram utilizados filtros de Butterworth com largura de faixa de desvanecimento $\mathrm{B}_{\mathrm{d}}$, da ordem de 10 a 30 por cento da taxa de sinalização. Estes valores correspondem a desvanecimentos cuja variação de amplitude ao longo do intervalo de sinalização é lenta.

Os valores dos parâmetros do modelo $\left(\mu_{0}, b_{0}, d_{0}\right)$ são obtidos usando-se resultados de medidas experimentais que produzam melhor ajuste para cada situação caracterizada por atributos como, por exemplo: ângulo de elevação do satélite, área rural ou urbana, densi- 
dade de vegetação (as referências [7,9] apresentam valores para estes parâmetros). $\mathrm{O}$ grau de atenuação sofrido pela componente de visada direta caracteriza três situações de desvanecimento: suave, médio, severo. O simulador de desvanecimento apresentado na Fig.3 apresenta uma boa concordância com o modelo teórico descrito pela expressão (5) [10].

A caracterização até aqui considerada (função distribuição de probabilidade da amplitude do sinal recebido) é uma estatística de primeira ordem. No simulador de desvanecimento da Fig.3, os filtros digitais controlam apenas as estatísticas de ordem superior à primeira (na literatura diversas possibilidades têm sido consideradas para a caracterização de tais estatísticas, por exemplo, fdp da taxa de variação da amplitude, fdp da taxa de variação da fase) e não interferem nas estatísticas de primeira ordem. Em [8], foi mencionado que com filtros Butterworth de três pólos obtém-se um bom ajuste às medidas experimentais disponíveis.

\subsection{Modulador CPM}

O modulador CPM realiza um mapa da sequiência de símbolos $\alpha$ em um conjunto de sinais que possuem envoltória constante e fase contínua. A expressão geral do sinal modulado $s\left(t, \underline{\alpha}_{k}\right)$, no intervalo $\tau_{\mathrm{k}}$ é dada por:

$$
\mathrm{s}\left(\mathrm{t}, \underline{\alpha_{k}}\right)=\Re e\left[\widetilde{s}\left(\mathrm{t}, \underline{\alpha}_{\mathrm{k}}\right) \mathrm{e}^{\mathrm{j} 2 \pi \mathrm{f}_{\mathrm{c}} \mathrm{t}}\right] \quad ; \quad \mathrm{t} \in \tau_{\mathrm{k}}
$$

O operador $\Re$ e significa a parte real de uma função complexa. A envoltória complexa do sinal modulador $\widetilde{\mathbf{s}}\left(\mathrm{t}, \underline{\alpha}_{\mathrm{k}}\right)$ tem a seguinte expressão:

$$
\widetilde{\mathrm{s}}\left(\mathrm{t}, \underline{\alpha_{k}}\right)=\sqrt{2 \mathrm{E}_{\mathfrak{S}} / \mathrm{T}} \mathrm{e}^{\phi\left(\mathrm{t}, \underline{\alpha}_{\mathrm{k}}\right)} \quad ; \mathrm{t} \in \tau_{\mathrm{k}}
$$

$O$ vetor $\underline{\alpha}_{k}$ especifica a sequiência de símbolos formada pelo símbolo $\alpha_{k}$ correspondente ao símbolo transmitido no intervalo $\tau_{\mathrm{k}}$ e todos os demais símbolos precedentes, ou seja, $\underline{\alpha}_{k}=\left(\ldots, \alpha_{k-2}, \alpha_{k-1}, \alpha_{k}\right)$. E é a energia por símbolo do sinal transmitido, $f_{c}$ é a freqüência da portadora e $\phi\left(t, \underline{\alpha}_{k}\right)$ é a fase do sinal cuja expressão geral é da forma

$$
\phi\left(t, \underline{\alpha_{k}}\right)=2 \pi \mathrm{h} \int_{0}^{\mathrm{t}} \sum_{\mathrm{i} \leq \mathrm{k}} \alpha_{\mathrm{i} g}(\tau-\mathrm{i} T) \mathrm{d} \tau+\phi_{0} \quad: \mathrm{t} \in \tau_{\mathrm{k}}
$$

Em (9) $\mathrm{g}(\mathrm{t})$ (denominado pulso de frequiência) é um pulso com formato arbitrário no intervalo $0 \leq \mathrm{t} \leq \mathrm{LT}$ (não contendo impulsos, pois $\phi\left(\mathrm{t}, \underline{\alpha}_{\mathrm{k}}\right)$ é uma função contínua no tempo) e identicamente nulo fora deste intervalo, h é o índice de modulação e $\phi_{0}$ é uma fase 
constante arbitrária que sem perda de generalidade pode ser igual a zero no caso coerente. O parâmetro L é denominado comprimento de correlação do pulso g(t). Definindo a função:

$$
\mathrm{q}(\mathrm{t})=\int_{0}^{\mathrm{t}} \mathrm{g}(\tau) \mathrm{d} \tau \quad ;-\infty<\mathrm{t}<\infty
$$

a fase do sinal em (9), pode ser reescrita da seguinte forma:

$$
\phi\left(t, \alpha_{k}\right)=2 \pi h \sum_{i \leq k} \alpha_{i} q(t-i T)+\phi_{0} \quad ; t \in \tau_{k}
$$

Observe que a fase $\phi\left(t, \underline{\alpha}_{k}\right)$ é uma função linear dos símbolos $\alpha_{i}$. A função $q(t)$ definida em (10) é denominada pulso resposta de fase. Tendo em vista que o pulso $q(t)$ tem, em geral, duração infinita, a fase do sinal modulado em um dado intervalo $\tau_{\mathrm{k}}$ não depende apenas do símbolo corrente $\alpha_{\mathrm{k}}$, mas também de todos os símbolos anteriores. Existe, então, uma memória infinita no sinal transmitido.

Dois formatos de pulso serão considerados neste trabalho: Pulso Retangular (L-REC) e o Pulso Cosseno Levantado (L-RC). Quando $L=1$, a modulação CPM é designada CPM com resposta plena, enquanto $L>1$ caracteriza a modulação CPM com resposta parcial. As expressões do pulso $g(t)$ no intervalo $[0, \mathrm{LT}]$ são dadas a seguir:

$$
\begin{gathered}
\mathrm{L}-\mathrm{REC}: \mathrm{g}(\mathrm{t})=1 / 2 \mathrm{LT} \\
\mathrm{L}-\mathrm{RC}: \mathrm{g}(\mathrm{t})=1 / 2 \mathrm{~L}][1-\cos (2 \pi \mathrm{t} / \mathrm{LT})]
\end{gathered}
$$

O pulso $g(t)$ apresenta a seguinte normalização:

$$
\int_{0}^{L T} g(\tau) d \tau=1 / 2
$$

Como o pulso $\mathrm{g}(\mathrm{t})$ é nulo fora do intervalo $[0, \mathrm{LT}]$, conclui-se que o pulso $\mathrm{q}(\mathrm{t})$ apresenta a seguinte característica:

$$
\begin{array}{cc}
q(t)=0 & t \leq 0 \\
q(t)=1 / 2 & t \geq L T
\end{array}
$$

Para desenvolver uma descrição de estados para o modulador CPM, a expressão geral da fase $\phi\left(t, \alpha_{k}\right)$ pode ser reescrita considerando-se (13) e (14). Observa-se na expressão (11) 
'e no intervalo $\tau_{k}$, a função $q(t-i T)$ alcança o seu valor final igual a $1 / 2$ para $: \mathrm{k}-\mathrm{L}+1$. Define-se

$$
\phi\left(t, \underline{\alpha}_{k}\right)=\theta\left(t, \underline{\alpha}_{k}\right)+\theta_{k}
$$

Ide

$$
\begin{gathered}
\theta\left(\mathrm{t}, \underline{\alpha_{k}}\right)=2 \pi \mathrm{h} \sum_{\mathrm{i}=\mathrm{k}-\mathrm{L}+1}^{\mathrm{k}} \alpha_{\mathrm{i}} \mathrm{q}(\mathrm{t}-\mathrm{iT}) \\
\theta_{\mathrm{k}}=\pi \mathrm{h} \sum_{\mathrm{i}=-\infty}^{\mathrm{k}-\mathrm{L}} \alpha_{\mathrm{i}}
\end{gathered}
$$

- fase constante $\theta_{\mathrm{k}}$ denominada estado de fase representa a contribuição dos símbolos assados, cuja função $\mathrm{q}(\mathrm{t}$ ) alcançou o seu valor final. Como é de interesse um número inito de estados de fase, deve-se considerar índices de modulação racionais da forma $1=2 p_{1} / p_{2}$, onde $p_{1}$ e $p_{2}$ são primos entre si. Os possíveis estados de fase pertencem ao onjunto:

$$
0, \frac{2 \pi}{\mathrm{p}_{2}}, \frac{4 \pi}{\mathrm{p}_{2}}, \ldots, \frac{\left(\mathrm{p}_{2}-1\right) 2 \pi}{\mathrm{p}_{2}}
$$

O número de estados de fase distintos, p, é igual a $p_{2}$. De acordo com (15 e 16), a fase $\phi\left(t, \underline{\alpha}_{k}\right)$, no intervalo $\tau_{k}$. é completamente especificada pelo símbolo transmitido $\alpha_{k}$, por ( $\mathrm{L}$ - 1) símbolos passados $\alpha_{k-1} \ldots, \alpha_{k-L+1}$ e pelo estado de fase. $O$ estado do modulador no intervalo $\tau_{\mathrm{k}}$ pode ser definido pelo vetor $\underline{\sigma}_{k}$ dado por:

$$
\underline{\sigma}_{k}=\left(\theta_{k}, \alpha_{k-1}, \ldots, \alpha_{k-L+1}\right)
$$

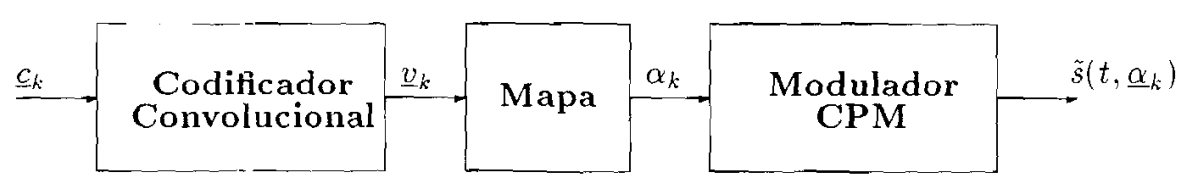

Figura 4 - Diagrama para o esquema Codificador/Modulador combinados

74 
Is $(\mathrm{L}-1)$ símbolos $\alpha_{\mathrm{k}-1}, \ldots, \alpha_{\mathrm{k}-\mathrm{L}+1}$ definem o estado correlativo do modulador. O núnero de estados distintos é igual a $\mathrm{pQ}^{\mathrm{L}-1}$.

) símbolo corrente $\alpha_{\mathrm{k}}$ determina a transição do estado corrente $\underline{\sigma}_{\mathrm{k}}$ para o próximo estado $\tilde{s}_{-k+1}$ e univocamente define a fase transmitida no intervalo. O estado $\underline{\sigma}_{k+1}$ é descrito pelo 'etor $\underline{\sigma}_{k+1}=\left(\theta_{k+1}, \ldots \alpha_{k}, \ldots, \alpha_{k-L+2}\right)$. A partir da expressão (17), uma relação recursiva ıode ser obtida para o cálculo do próximo estado de fase $\theta_{\mathrm{k}+1}$ dada por ${ }_{\mathrm{k}+1}=\theta_{\mathrm{k}}+\pi \mathrm{h} \alpha_{\mathrm{k}-\mathrm{L}+1}$.

) número de estados de fase efetivo é reduzido a metade se o valor de $\mathrm{p}_{2}$ for par. Neste aso, se em algum intervalo o estado de fase pertence ao subconjunto $\left.i_{1}=0,4 \pi / \mathrm{p}_{2}, \ldots,\left(\mathrm{p}_{2}-2\right) 2 \pi / \mathrm{p}_{2}\right\}$ no próximo intervalo pertencerá ao subconjunto $\left.i_{2}=2 \pi / \mathrm{p}_{2}, \ldots,\left(\mathrm{p}_{2}-1\right) 2 \pi / \mathrm{p}_{2}\right)^{\prime}$

\subsection{Descrição de Estado Conjunta para o CC/CPM}

\ Fig.4 representa o esquema Codificador/Modulador CC/CPM. Nesta seção investigaemos uma descrição de estado conjunta para este código concatenado.

) estado do sistema da Fig.4, no intervalo $\tau_{\mathrm{k}}$, corresponde à informação necessária para jue, dado o vetor de informação $\underline{c}_{k}$, o sinal $\tilde{s}\left(t, \underline{\alpha}_{k}\right)$ seja completamente especificado. $\mathrm{Na}$ specificação deste estado conjunto, deve ser levado em consideração o estado do moduador (descrito pelo vetor $\sigma_{\mathrm{k}}$ em (19)), a memória do codificador convolucional $v$, e o ato dos símbolos $\alpha_{i}$, pertencentes ao conjunto $\left\{\alpha_{i}\right\}_{i=k-1}^{k-L+1}$, não serem independentes.

Jenericamente o símbolo $\alpha_{\mathrm{k}}$ na entrada do modulador pode ser representado na forma:

$$
\alpha_{\mathrm{k}}=\mathcal{F}\left(\underline{\Omega_{\mathrm{k}}, \underline{\mathrm{C}} \mathrm{k}}\right)
$$

Inde $\mathcal{F}$ descreve funcionalmente o codificador e o mapa. De forma análoga, o estado do zodificador convolucional no próximo intervalo pode ser representado através da função $\hat{\jmath}$, ou seja:

$$
\underline{\Omega_{\mathrm{k}}+1}=\underline{G}\left(\underline{\Omega_{\mathrm{k}}}, \underline{\mathrm{c}_{\mathrm{k}}}\right)
$$

Expressando o estado correlativo do modulador de acordo com a notação (20) e (21) obemos as relações:

Zevista da Sociedade Brasileira de Telecomunicações 


$$
\begin{aligned}
& \alpha_{\mathrm{k}-\mathrm{L}+1}=\mathcal{F}\left(\underline{\Omega}_{\mathrm{k}-\mathrm{L}+1}, \underline{\mathrm{c}}_{\mathrm{k}-\mathrm{L}+1}\right) \\
& \alpha_{k-L+2}=\mathcal{F}\left(\underline{\Omega}_{k}-\mathrm{L}+2, \underline{c}_{k}-\mathrm{L}+2\right) \\
& =\mathcal{F}\left(\underline{G}\left(\underline{\Omega}_{\mathrm{k}}-\mathrm{L}+1, \underline{\mathrm{c}} \mathrm{k}-\mathrm{L}+1\right), \underline{\mathrm{c}} \mathrm{k}-\mathrm{L}+2\right) \\
& =U(\underline{\Omega} \mathrm{k}-\mathrm{L}+1, \underline{\mathrm{c}} \mathrm{k}-\mathrm{L}+1, \underline{\mathrm{c}} \mathrm{k}-\mathrm{L}+2)
\end{aligned}
$$

O relacionamento recursivo acima pode ser estendido até a especificação do símbolo $\alpha_{k-1}$, concluindo-se que o estado correlativo do modulador CPM no intervalo $\tau_{k}$ é completamente especificado pelo estado do codificador $\Omega_{k-L+1}$ e por $\mathrm{L}-1$ vetores $\underline{c}_{k-L+1}, \ldots \underline{c}_{k}$. De forma análoga, pode-se concluir que estes dados também especifícam o estado do codificador $\Omega_{\mathrm{k}}$.

Então, um conjunto de $v+\varphi(L-1)$ bits, o estado de fase $\varphi_{k}$, juntamente com o vetor $c_{k}$, permitem uma descrição sem ambiguidade do sinal modulado em $\tau_{\mathrm{k}}$.

Define-se, portanto, o estado do sistema codificador/modulador da Fig.4, no intervalo $\tau_{k}$, pelo vetor $\sigma_{k}^{*}$, descrito na seguinte forma:

$$
\underline{\sigma}_{\mathrm{k}}^{*}=\left(\theta_{\mathrm{k}}, \underline{\Omega}_{\mathrm{k}-\mathrm{L}+1}, \underline{\mathrm{c}}_{\mathrm{k}-1}, \ldots, \underline{\mathrm{c}}_{\mathrm{k}}-\mathrm{L}+1\right)
$$

Usando a relação (1), pode-se expressar $\underline{\sigma}_{k}^{*}$ como um vetor composto pelo estado de fase e por $v+l(L-1)$ bits independentes da forma:

$$
\underline{\sigma}_{k}^{*}=\left(\theta_{k}, \Omega_{k}^{1}-\mathrm{L}+1, \ldots, \Omega_{k}^{v}-\mathrm{L}+1, b_{k}-1, \ldots, b_{k}-1(L+1)\right)
$$

O número de estados distintos $\left(\mathrm{N}_{\sigma}\right)$ é igual a:

$$
\mathrm{N}_{\sigma}=\mathrm{p} 2^{v_{2}} 2^{\mathrm{l}(\mathrm{L}-\mathrm{l})}
$$

A partir da descrição de estados em (24), pode-se associar uma treliça de estados ao sistema CC/CPM. Cada nó da treliça está associado a um estado e toda seqüência de vetores de informação $\left\{\underline{c}_{k}\right\}$ está associada biunivocamente a um percurso na treliça. 


\section{Distância Euclidiana Mínima para CC/CPM}

Nesta seção são apresentados esquemas CC/CPM que produzem melhores valores de $\mathrm{d}_{\text {min }}^{2}$ para sistemas CC/CPM. O codificador convolucional utilizado tem uma estrutura realimentada. A seguir apresentamos a expressão de $\mathrm{d}_{\min }^{2}$ para o sistema CC/CPM.

Considere uma sequiência de vetores $\underline{\mathrm{c}}^{\alpha}=\left(\underline{\mathrm{c}}_{0}^{\alpha}, \underline{\mathrm{c}}_{1}^{\alpha}, \ldots, \mathrm{c}_{\mathrm{N}}^{\alpha}\right)$ composta de $\mathrm{N}$ vetores à entrada do codificador convolucional, conforme o esquema da Fig.4. Para um dado estado $\Omega_{0}, \underline{c}^{\alpha}$, produzirá a sequiência $\underline{\alpha}=\left(\alpha_{0}, \ldots, \alpha_{N}\right)$ composta de $\mathrm{N}$ símbolos que serão transmitidos pelo modulador no intervalo $0 \leq \mathrm{t} \leq \mathrm{NT}$. O sinal modulado, $\mathrm{s}_{\alpha}(\mathrm{t})$, associado a sequiência $\alpha$ de comprimento $N$ é dado por:

$$
\mathrm{s}_{\alpha}(\mathrm{t})=\sum_{\mathrm{k}=0}^{\mathrm{N}-1} \mathrm{~s}\left(\mathrm{t}, \underline{\alpha}_{\mathrm{k}}\right)
$$

onde s(t, $\left.\underline{\alpha}_{\mathrm{k}}\right)$ é dado em (17). O quadrado da distância euclidiana entre dois sinais, $\mathrm{s}_{\alpha}(\mathrm{t}) \mathrm{e}$ $\mathrm{s}_{\beta}(\mathrm{t})$ é definido por:

$$
D_{N}^{2}(\underline{\alpha}, \underline{\beta})=\int_{0}^{N T}\left[s_{\alpha}(t)-s \beta(t)\right]^{2} d t
$$

Define-se a distância euclidiana quadrática mínima normalizada, $\mathrm{d}_{\mathrm{min}}^{2}$, para o sistema CC/CPM da seguinte forma:

$$
\mathrm{d}_{\min }^{2}=\lim _{\mathrm{N} \rightarrow \infty}\left\{\frac{1}{2 \mathrm{E}_{\mathrm{b}}} \mathrm{D}_{\min , \mathrm{N}}^{2}\right\}
$$

onde

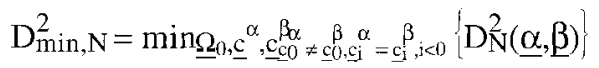

$\mathrm{D}_{\min , \mathrm{N}}$ é a distância euclidiana mínima no intervalo [0,NT]. Na expressão (28) a distância euclidiana deve ser normalizada em relação $a \mathrm{E}_{\mathrm{b}}$ (energia por bit de informação) a fim de que sistemas com alfabetos de diferentes tamanhos sejam comparados em igualdade de condições (mesma potência média e taxa de informação). No cálculo de $\mathrm{D}_{\min , \mathrm{N}}$ todos 
os pares de trajetórias de fase que divergem de um nó comum em $\mathrm{t}=0$ (equivale a condição $\left.\underline{c}_{0}^{\alpha} \neq \underline{c}_{0}^{\beta}, c_{-}^{\alpha}=\underline{c}_{1}^{\beta}, \mathrm{i}<0\right)$ devem ser considerados na equação (27) e a minimização é realizada para todos os pares de sequiências $\underline{\alpha}$, $\beta$ e para o estado inicial do codificador $\Omega_{0}$.

Usando (26) e (7) em (27) e considerando que $f_{c} T>>1, D_{N}^{2}(\underline{\alpha}, \underline{\beta})$ pode ser expressa na forma:

$$
D_{N}^{2}(\underline{\alpha}, \underline{\beta})=2 \mathrm{E}_{\mathrm{s}}\left[\mathrm{N}-\frac{1}{\mathrm{~T}} \int_{0}^{\mathrm{NT}} \cos \left[\Delta \phi\left(\mathrm{t}, \underline{\gamma k}_{\mathrm{k}}\right)\right] \mathrm{dt}\right]
$$

onde $\Delta \phi\left(t, \gamma_{k}\right)$ é a diferença de fase entre as sequiências $\underline{\alpha}, \underline{\beta}$ definida por:

$$
\Delta \phi\left(\mathrm{t}, \gamma_{\mathrm{k}}\right)=2 \pi \mathrm{h} \sum_{i \leq k} \gamma_{i} \mathrm{q}(\mathrm{t}-\mathrm{iT})
$$

com

$$
\underline{\alpha}=\underline{\alpha}_{k}-\underline{\beta} \mathrm{k}
$$

Para o sistema CC/CPM a energia por bit de informação é $\mathrm{E}_{\mathrm{b}}=\mathrm{E}_{\mathrm{s}} / \ell$ ( $\ell$ é o numerador da taxa do código convolucional). A expressão para a distância mínima pode ser obtida a partir de (28), considerando (29) e (30), e é expressa por:

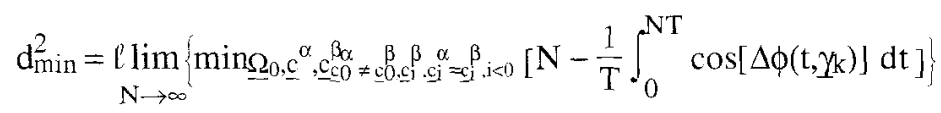

\subsection{Busca do Código com Máxima Distância Euclidiana Mínima}

Nesta seção são apresentados os resultados da busca da combinação CC/CPM com máxima distância euclidiana quadrática mínima. Para um dado sistema $C P M$ e para $v, R_{c} e$ mapas fixos, obtém-se (a partir de uma busca exaustiva) os polinômios geradores do codificador convolucional $\left.{ }^{\{} \mathrm{H}^{\mathrm{j}}(\mathrm{D})\right\}_{\mathrm{j}=0}^{\mathrm{m}}$, que maximiza a distância euclidiana quadrática mínima. Os códigos foram obtidos com o algoritmo de busca publicado por Mulligan e Wilson [4]. 
Os resultados obtidos são apresentados no apêndice A (Tab.1 até 8). É importante enfatizar, como referência ao consultar as tabelas, que o valor de $\mathrm{d}_{\min }^{2}$ para as modulações QPSK e MSK é igual a 2. As tabelas mostram valores de $\mathrm{N}_{\text {min }}$, nível a partir do qual todos os pares de sinais têm distância euclidiana maior que $\mathrm{d}_{\min }^{2}$. Os valores de $\mathrm{h}^{\mathrm{i}}$, que indicam os polinômios geradores do código convolucional que produziu o valor máximo de $\mathrm{d}_{\min }^{2}$ correspondem em notação decimal, ao vetor formado pelos coeficientes dos polinômios geradores, ou seja, $h^{i}=h_{v-1}^{i} 2^{v-2}+\ldots+h_{2}^{i} 2+h_{1}^{i}$.

Valores de $\mathrm{d}_{\min }^{2}$ para os melhores códigos correspondendo ao sistema CPM quaternário (definimos a notação $4 \mathrm{CPM}$ ) e codificador convolucional com taxa $R_{c}=1 / 2$ são apresentados nas Tabs.1 e 2. As tabelas mostram que os valores de $\mathrm{d}_{\min }^{2}$ crescem monotonicamente até aproximadamente $h=1 / 4$. Valores maiores de h, além denãoproduzir um aumentoded $\mathrm{d}_{\text {min }}^{2}$, correspondem a sinais CC/CPM com maior ocupação de faixa. Nesta região $(\mathrm{h}<1 / 4)$ um mesmo conjunto de polinômios geradores é obtido nos diversos casos. Verifica-se que, para um dado h, o aumento da memória $v$ melhora a eficiência em termos da energia consumida pelo sistema.

As Tabs.3 4 apresentam os valores de $\mathrm{d}_{\mathrm{min}}^{2}$ para osistema $4 \mathrm{CPM}, 1-\mathrm{RC}, \mathrm{R}_{\mathrm{c}}=1 / 2$. Para $v=2$, os valores da distância euclidiana são maiores quando comparados com os da Tab.1. Para $v=3$ este comportamento é verificado para $\mathrm{h}<1 / 4$.

Os melhores códigos para sistemas $4 \mathrm{CPM}, \mathrm{R}_{\mathrm{c}}=1 / 2, v=2$, com resposta parcial, são mostrados nas Tabs.5 e 6. Para valores pequenos de $\mathrm{h}(\mathrm{h} \leq 1 / 4)$, devido a memória existente na fase do sinal CPM, a sequiência que produz a distância euclidiana mínima é menos espaçada quando comparada a sinais com resposta plena, de modo que os valores de $\mathrm{d}_{\min }^{2}$ encontrados são menores quando comparados com os valores das Tabs.1 e 3. Em geral, o sistema 2-REC apresenta os menores valores de $\mathrm{d}_{\min }^{2}$ e maiores valores de $\mathrm{N}_{\min }$.

As Tabs.7 e 8 apresentam os resultados para o sistema $8 \mathrm{CPM}, \mathrm{R}_{\mathrm{c}}=2 / 3, \mathrm{v}=2$. Na Tab.7 $(\tilde{\mathrm{m}}=1)$ para alguns valores de índice de modulação $(\mathrm{h}=1 / 4, \mathrm{~h}=1 / 2)$ o par de trajetórias que produzem a distância mínima apresenta a característica de transição paralela, produzindo valores pequenos de $\mathrm{d}_{\min }^{2}$. Os melhores códigos com $\tilde{\mathrm{m}}=2$ são apresentados na Tab.8.

Sistemas CC/CPM com máximo valor $d e \mathrm{~d}_{\mathrm{min}}^{2}$, para o codificador convolucional com estrutura feedforward, foram obtidos por Ho e McLane [2] e Pizzi e Wilson [3]. Em [10] é realizada uma comparação entre os valores de $\mathrm{d}_{\min }^{2}$ para o codificador convolucional com estrutura realimentada com os resultados de [2] e [3]. Constata-se que a estrutura

Revista da Sociedade Brasileira de Telecomunicações 
salimentada produz maiores valores de distância mínima para os vários valores de índie de modulação (na comparação entre as duas estruturas, o codificador tem o mesmo varr deve $R_{c}$ ).

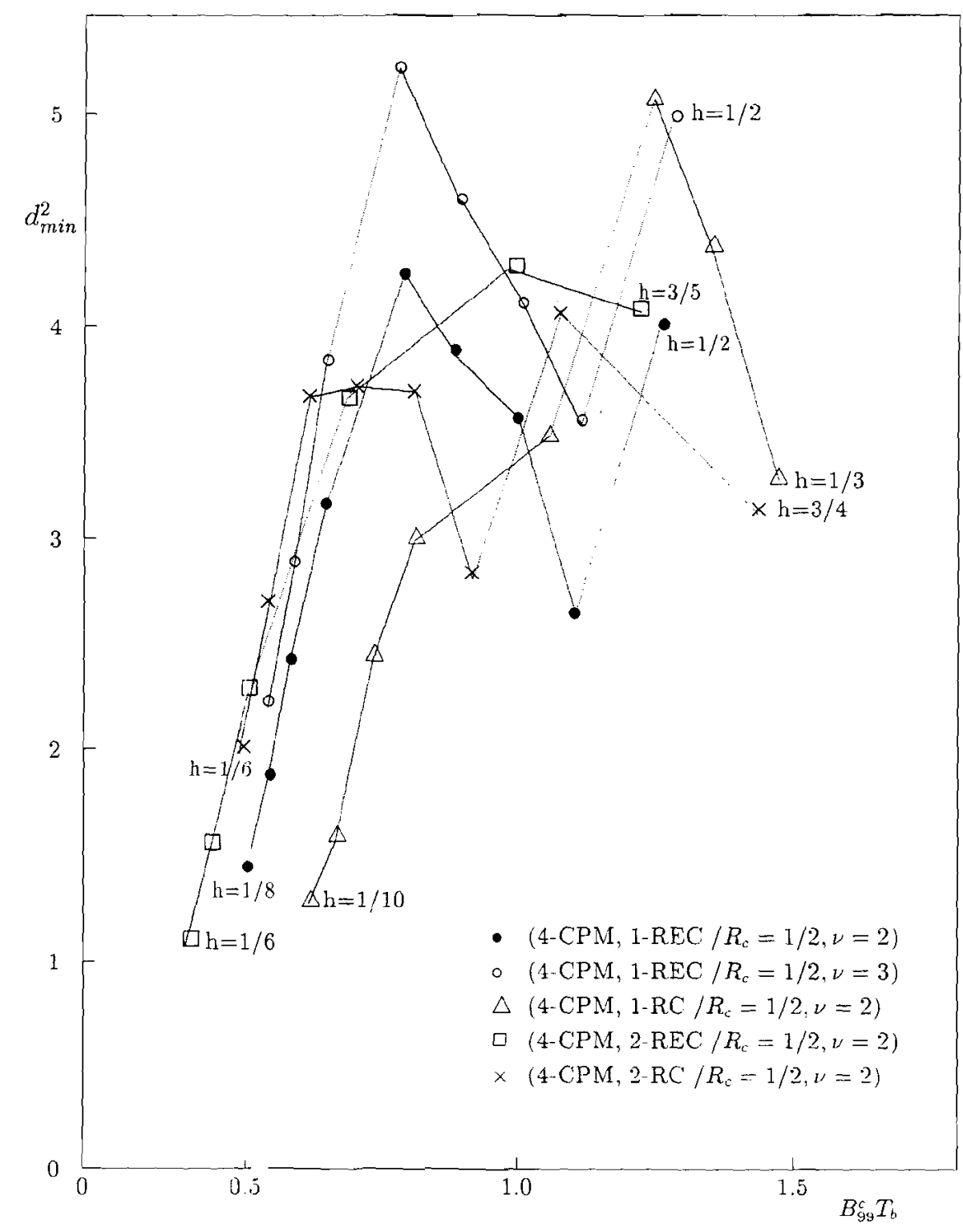

Figura 5 - Curvas de $d_{\min }^{2}$ versus $B_{99}^{C}$ para sistemas CC/CPM. $\tilde{m}=1$ 


\subsection{Curvas $d_{\min }^{2}$ versus Largura de Faixa}

Nesta seção os sistemas CC/CPM apresentados na seção 3.1 são comparados através de curvas no plano $\mathrm{d}_{\min }^{2}$ - largura de faixa. Estas curvas representam melhor o compromisso existente entre o aumento da distância mínima e a expansão de faixa produzida pela codificação convolucional.

A largura de faixa, $\mathrm{B}_{99}^{\mathrm{c}}$, adotada neste trabalho é definida como a banda de frequiências em que $99 \%$ da potência da envoltória complexa do sinal esteja compreendida entre as frequiências $\left[-\mathrm{B}_{99}^{\mathrm{c}}, \mathrm{B}_{99}^{\mathrm{c}}\right]$. Para o sistema $\mathrm{CC} / \mathrm{CPM}$ um resultado aproximado pode ser obtido considerando que os bits de saída do codificador convolucional são independentes. A largura de faixa normalizada, $\mathrm{B}_{99}^{\mathrm{c}} \mathrm{T}_{\mathrm{b}}$, é calculada a partir da expressão da largura de faixa normalizada de um sistema CPM puro $2^{\mathrm{m}+1}$-ário (cuja expressão está desenvolvida em [11]), normalizada pela taxa do codificador convolucional $R_{c}$. Em [3] comparou-se a Densidade Espectral de Potência (DEP) para sistemas CC/CPM obtidos por simulação, e a DEP obtida conforme descrito acima, obtendo-se uma boa concordância. A expressão exata para a DEP de sistemas CPM com símbolos de entrada previamente codificados está desenvolvida em [12].

Uma outra definição para a largura de faixa é denominada $B_{99,9}^{c}$. Neste caso, 99,9\% da potência do sinal está compreendida na faixa $\left[-\mathrm{B}_{99,9}^{\mathrm{c}}, \mathrm{B}_{99,9}^{\mathrm{c}}\right]$. Esta definiç̧ão abrange mais significativamente os lóbulos secundários do espectro de potência e deve ser considerada quando pretende-se considerar interferências em canais adjacentes.

A Fig.5 apresenta a curva $d_{\text {min }}^{2}$ versus $B_{99}^{c} T_{b}$. $O$ índice de modulação é variado ao longo da curva (decresce da direita para a esquerda) e seus valores extremos são indicados na figura. Na região de maior eficiência espectral (valores pequenos de h) o sistema 1-REC apresenta um melhor desempenho conjunto potência-largura de faixa (para uma mesma ocupação de banda, o sistema apresenta valores maiores de $\mathrm{d}_{\mathrm{min}}^{2}$ ) quando comparado com o sistema 1-RC. Nesta região de interesse prático (aproximadamente $B_{99}^{c} T_{b} \leq 1$ ) o sistema 2-REC apresenta um desempenho superior dentre os quatro sistemas comparados na figura.

A Fig.6 apresenta a curva $d_{\min }^{2}$ versus $B_{99,9}^{c} T_{b}$. As conclusões referentes à Fig.5 também são válidas para os sistemas com resposta plena. Neste caso, em que a banda de $99,9 \%$ é considerada, o sistema 2-RC apresenta o melhor desempenho na região de maior eficiência espectral. 


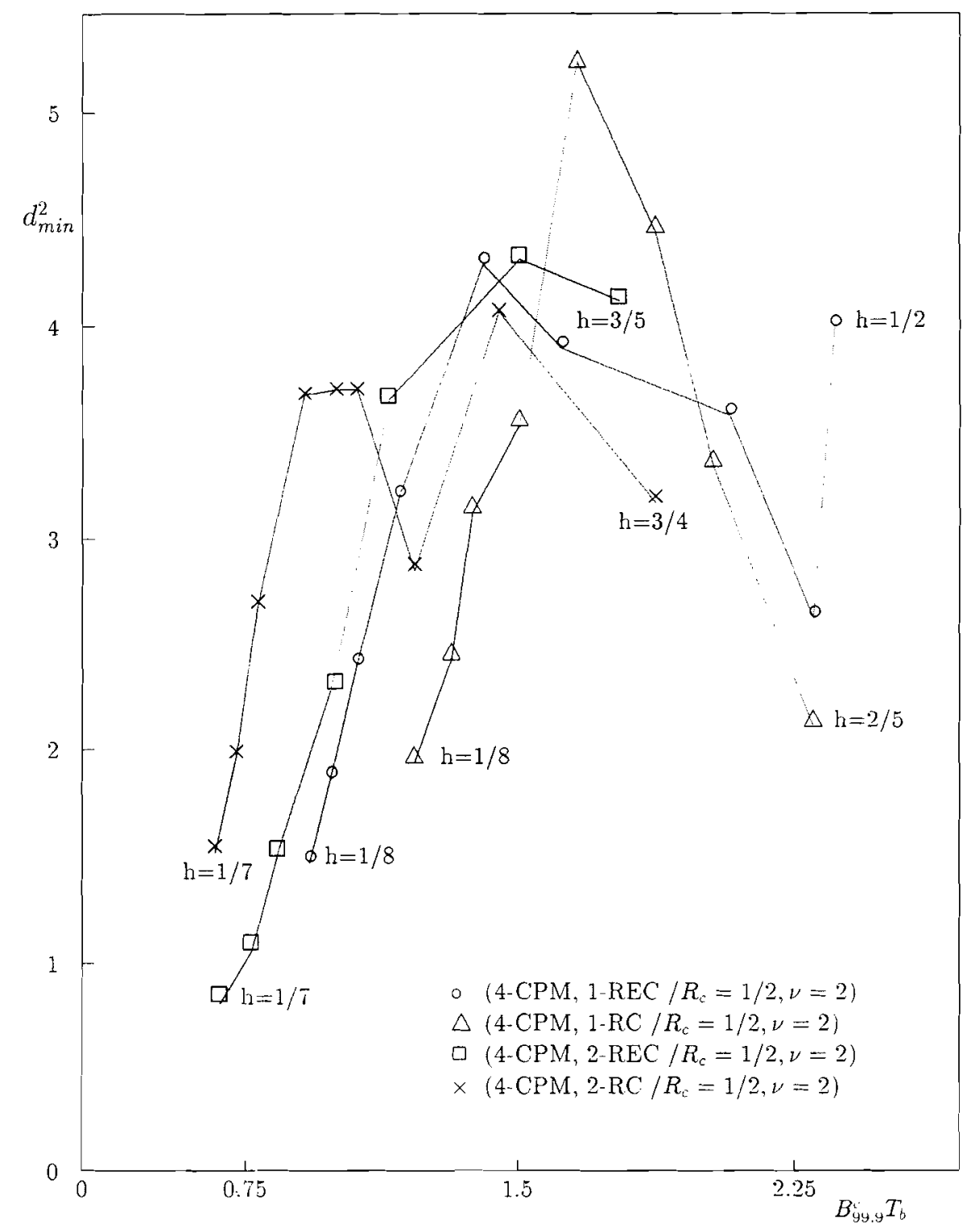

Figura 6 - Curvas de $d_{\min }^{2}$ versus $B_{99,9}^{c}$ para sistemas CC/CPM. $\tilde{m}=1$ 


\section{Desempenho do Sistema CC/CPM Usando o Algoritmo-M de Decodificação}

Esta seção trata da análise da decodificação de sistemas CC/CPM para um decodificador subótimo em que o conjunto de percursos onde é realizada a busca da sequiência transmitida é limitado. A decodificação com o Algoritmo-M deve apresentar um esforço computacional e um requisito de memória reduzidos com relação ao esforço requerido pelo Algoritmo de Viterbi. Uma característica desejável é que para razão sinal ruído elevada o desempenho do decodificador subótimo seja próximo ao desempenho alcançado pelo Algoritmo de Viterbi. Nesta seção analisa-se o desempenho do decodificador baseado no Algoritmo-M e mostra-se que este apresenta as propriedades mencionadas acima.

Uma breve descrição do Algoritmo-M de decodificação é dada a seguir. O Algoritmo-M (AM) opera de forma similar ao Algoritmo de Viterbi (AV). A diferença básica é que no AM, para cada nível da treliça de decodificação apenas os M percursos com maiores métricas são considerados como sobreviventes, enquanto que no AV o número de sobreviventes é igual ao número total de estados do código, $\mathrm{N}_{\sigma}$. Maiores detalhes sobre o AM são apresentados nas referências $[13,14]$.

Para a seqüência $\underline{\alpha}_{\mathrm{k}}$, a métrica $\mathrm{J}\left(\underline{\alpha}_{\mathrm{k}}\right)$ é definida como a correlação entre o sinal recebido $r(t)$ até o intervalo $\tau_{k}$ e o sinal correspondente a esta sequiência, $s\left(t, \underline{\alpha}_{k}\right)$. Sua expressão é dada por

$$
\left.\mathrm{J}\left(\underline{\alpha}_{k}\right)=\int_{-\infty}^{\left(k_{-}+1\right) \mathrm{T}} r(t) s\left(t, \underline{\alpha}_{k}\right) d t\right]
$$

Os $M$ sobreviventes no intervalo $\tau_{\mathrm{k}+1}$ são usados pelo AM para selecionar o símbolo de informação $\hat{\underline{c}}_{\mathrm{k}-\mathrm{L}_{\mathrm{d}}}$, associado ao percurso de maior métrica, como sendo o símbolo decodificado. O parâmetro $L_{d}$ é denominado de profundidade de decisão do Algoritmo-M.

O AM não é um algoritmo de busca exaustiva (alguns percursos com menor probabilidade de serem o percurso transmitido são descartados ao longo da busca na treliça). Neste caso o esforço computacional e a memória requerida são constantes em cada intervalo e proporcionais a $M$. Para $M=N_{\sigma}$ o AM corresponde ao AV (a busca torna-se exaustiva). 


\subsection{Simulação de CC/CPM usando o Algoritmo-M de Decodificação em Canais com Desvanecimento}

O desempenho de um sistem de comunicações móveis que utiliza o esquema CC/CPM e usa um decodificador baseado no Algoritmo-M é analisado através da simulação do sistema de comunicações apresentado na Fig.1.

A técnica utilizada para a análise de desempenho é a simulação em computador digital. As diversas formas de onda presentes no sistema são representadas por suas componentes em banda básica, através de suas amostras em instantes de tempo igualmente espaçadas. O número de amostras por intervalo de sinalização $\mathrm{N}_{\mathrm{a}}$ foi selecionado simulando o sistema para valores crescentes de $\mathrm{N}_{\mathrm{a}}$ até não ocorrer mudanças significativas na taxa de erro. Verificou-se que 10 amostras por intervalo, em cada canal, é uma escolha adequada para as simulações realizadas. Considerou-se o valor da profundidade de decisão, $\left(\mathrm{L}_{\mathrm{d}}\right)$, suficientemente alto para que não degrade o desempenho do sistema. Em todas as simulações o valor de $\mathrm{L}_{\mathrm{d}}$ é igual a 30 .

O modelo de simulação utilizado adota uma simplificação no que se refere a influência do desvanecimento sobre a fase do sinal recebido. Tendo em vista que não foi incorporado ao modelo um módulo de sincronização, supõe-se que não existe erro de fase introduzido pelo desvanecimento. A sincronização de fase e relógio, para eliminar estes erros introduzidos pelos filtros digitais é realizada de forma ideal [10].

O desempenho de dois códigos são examinados através de simulação. O nosso objetivo é avaliar a dependência do sistema com o parâmetro $M$ e poder estimá-lo para que o desempenho do AM seja próximo ao desempenho do decodificador ótimo.

As Figs.7 e 8 apresentam curvas de probabilidade de erro de bit, $P_{b}$, versus $M$ para vários valores de $E_{b} / N_{0}$. O código simulado é (4CPM,1-REC, $\left.R_{c}=1 / 2, v=2\right), h=1 / 5$, com $N_{\sigma}=20$ estados efetivos. Duas situações de desvanecimento são consideradas: suave e médio. As curvas para o caso de desvanecimento severo não serão apresentadas, devido ao fato que este caso apresenta valores de probabilidade de erro bastante elevados, para a faixa de $\mathrm{E}_{\mathrm{b}} / \mathrm{N}_{0}$ considerada.

As figuras mostram que para um dado valor de $\mathrm{E}_{\mathrm{b}} / \mathrm{N}_{0}, \mathrm{P}_{\mathrm{b}}$ decresce até um certo valor quando $\mathrm{M}$ aumenta (este é o valor final de $\mathrm{P}_{\mathrm{b}}$ obtido pelo $\mathrm{AV}$ ). Observa-se que o valor de $M$ para $P_{b}$ atingir o seu valor final decresce com o aumento de $E_{b} / N_{0}$. Para valores elevados de $E_{b} / N_{0}$ a complexidade requerida para o AM é substancialmente menor que a complexidade do AV, considerando o mesmo valor de desempenho. Esta observação mostra que o AV opera com uma complexidade desnecessária, corroborando o fato que o AM é mais eficiente que o AV.

84 
Em sistemas de comunicações binário que usam códigos convolucionais, foi provado analiticamente que o desempenho do AM é similar ao desempenho do AV, para valores de M significantemente menores que o número de estados do código [13]. Para sistemas CPM e CC/CPM, estes resultados não foram mostrados analiticamente, mas observados através de simulações.

Na implementação do AM, um sério problema que pode acontecer é a perda do percurso correto. Este fenômeno ocorre quando, no processo de decodificação, o percurso transmitido não faz parte dos $M$ percursos sobreviventes em um dado nível da treliça. Neste caso a busca prossegue de maneira desordenada ao longo da treliça e só o ruído pode recolocar o percurso correto entre os M sobreviventes. Lin [15] observou que o fenômeno de perda do percurso correto pelo AM ocorre em sistemas binários que usam códigos convolucionais. Aulin [16] constatou que a modulação CPM não apresenta esta característica, ou seja, quando o percurso correto não faz parte dos M percursos sobreviventes, $\mathrm{o}$ algoritmo rapidamente o recupera. A probabilidade de ocorrência da perda de percurso decresce com o aumento de $E_{\mathrm{b}} / \mathrm{N}_{0}$. Nas simulações realizadas com o CC/CPM não foi constatado o problema da perda do percurso correto.

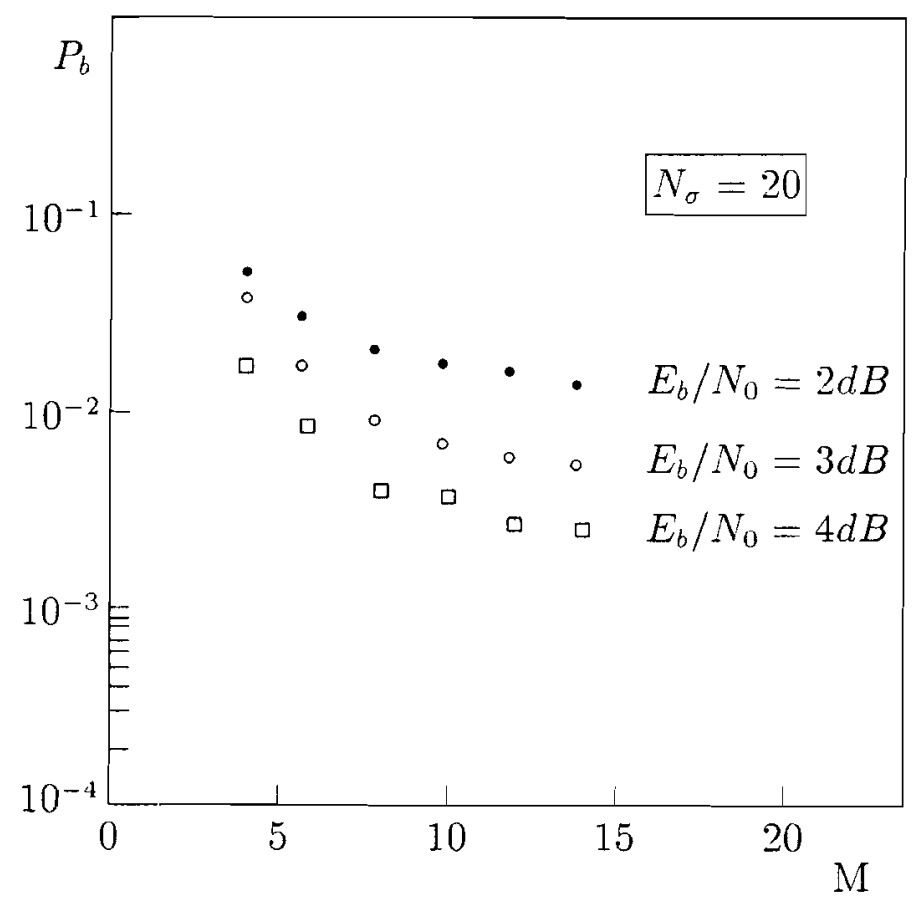

Figura 7 - Probabilidade de erro de bit versus $M\left(4 C P M, 1-R E C, R_{c}=1 / 2, v=2\right)$, $h=1 / 5, d_{\min }^{2}=3.19$. Desvanecimento suave. 


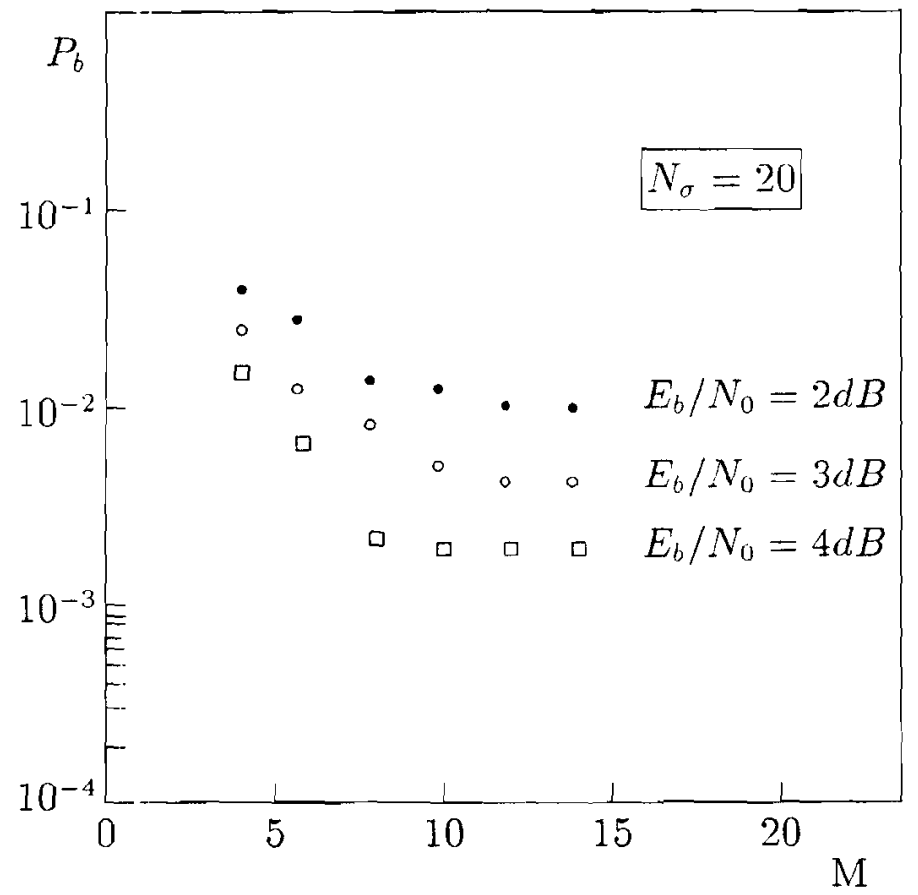

Figura 8 - Probabilidade de erro de bit versus $M\left(4 C P M, 1-R E C, R_{C}=1 / 2, v=2\right)$, $\mathrm{h}=1 / 5, \mathrm{~d}_{\min }^{2}=3.19$. Desvanecimento médio. 


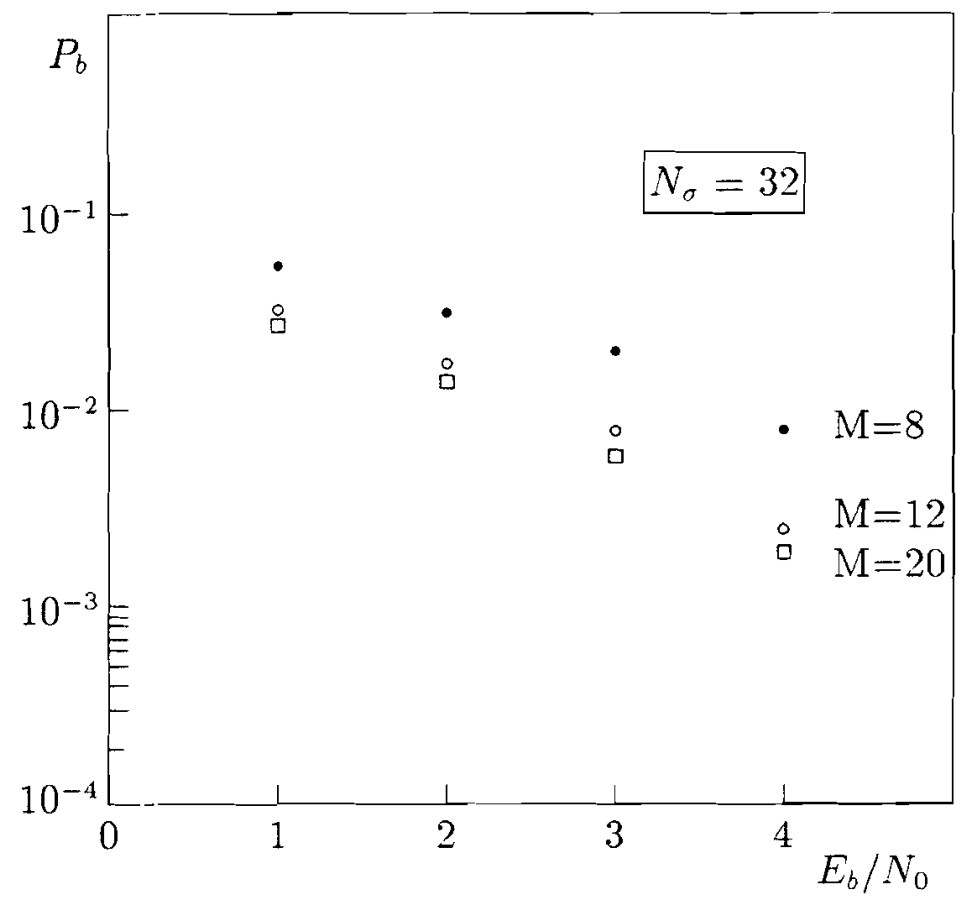

Figura 9 - Probabilidade de erro de bit versus (4CPM, 2-RC, $R_{C}=1 / 2, v=2$ ), $h=1 / 4, d_{\min }^{2}=3.72$. Desvanecimento suave.

As Figs.9 e 10 apresentam curvas $P_{b}$ versus $E_{b} / N_{0}$ para vários valores de $M$. O código simulado é (4CPM, 2-RC, $\left.\mathrm{R}_{\mathrm{c}}=1 / 2, v=2\right), \mathrm{h}=1 / 4$, com número de estados efetivos $\mathrm{N}_{\sigma}=32$. Para um dado valor de $\mathrm{P}_{\mathrm{b}}$ da ordem de $10^{-3}$, espera-se que o desempenho do AM para $\mathrm{M}=20$ atinja um valor próximo do seu valor final (desempenho igual ao AV). A Fig.9 (desvanecimento suave) mostra que, para $P_{b}$ da ordem de $10^{-3}$, e decodificação usando o $\mathrm{AM}$ para $\mathrm{M}=12$ obtém-se uma perda de aproximadamente $0.4 \mathrm{db}$ em relação ao desempenho para $M=20$. Para $M=8$ a perda é de aproximadamente $1 \mathrm{db}$. Quando se considera valores de $\mathrm{P}_{\mathrm{b}}$ inferiores a $10^{-3}$ a degradação de $\mathrm{E}_{\mathrm{b}} / \mathrm{N}_{0}$ observada é menor quando comparada com o obtido acima. Resultados semelhantes são obtidos a partir da curva apresentada na Fig.10 (desvanecimento médio).

Revista da Sociedade Brasileira de Telecomunicações 


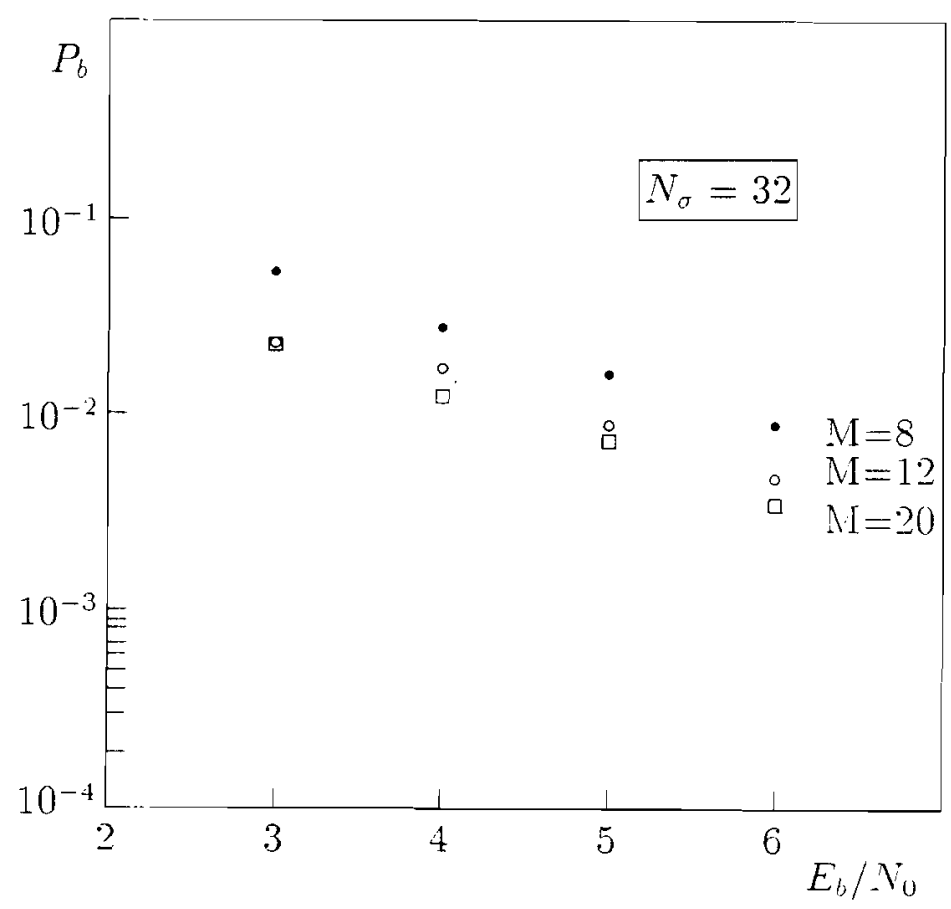

Figura 10 - Probabilidade de erro de bit versus $E_{b} / N_{0}$

(4CPM, 2-RC, $\left.R_{C}=1 / 2, v=2\right), h=1 / 4, d_{\min }^{2}=3.72$. Desvanecimento médio.

\section{i. Conclusão}

J presente trabalho desenvolveu um modelo para simular em tempo discreto um sistema le comunicações digital que permite analisar o desempenho de esquemas de modulação IC/CPM em um canal com desvanecimento, quando o esquema de decodificação é ba.eado no Algoritmo-M.

† simulação é realizada em computador digital e o modelo desenvolvido utiliza a repre,entação em banda básica discretizada no tempo, para representar os diversos sinais. $\mathrm{O}$ nódulo que simula o desvanecimento é implementado basicamente realizando-se a filragem de ruído gaussiano branco limitado em faixa. A estrutura do simulador de desvarecimento foi desenvolvida a partir de um modelo teórico que caracteriza um canal de :omunicações móveis via satélite. Este modelo apresenta resultados corroborados por nedidas experimentais [9].

A seleção do esquema CC/CPM ótimo é baseado no critério da máxima distância eucliliana quadrática mínima. Os resultados da busca de $\mathrm{d}_{\min }^{2}$ para códigos CC/CPM com nú- 
mero de estados pequenos são apresentados no apêndice A. Nestas tabelas são indicados os polinômios geradores do código convolucional com estrutura realimentada que maximizam $d_{\min }^{2}$ para um dado esquema CPM e para $v, R_{c}$ e o mapa fixos.

Para $v=2$, valores de $\mathrm{d}_{\min }^{2}$ maiores são obtidos para o sistema 1-RC, quando comparados com o sistema 1-REC. Em particular, $\mathrm{d}_{\text {min }}^{2}=5,15$ para $\mathrm{h}=1 / 4$ é um valor extremamente alto. $\mathrm{O}$ aumento de memória $v$ proporciona valores de $\mathrm{d}_{\min }^{2}$ mais elevados. Os resultados para sistemas CPM com resposta parcial para $v$, indicam que na região de alta eficiência espectral os valores de $\mathrm{d}_{\text {min }}^{2}$ são menores quando comparados com os valores para sistemas CPM resposta plena.

Os diversos esquemas são comparados sob a restrição de mesma ocupação de faixa, através de curvas $\mathrm{d}_{\mathrm{min}}^{2}$-largura de faixa. Constata-se que na região de maior eficiência espectral, os sistemas CPM com resposta parcial apresentam um desempenho superior.

Por fim, é realizada uma simulação da decodificação de sistemas CC/CPM usando o Algoritmo-M (AM) de decodificação em presença de desvanecimento. Neste algoritmo o número de percursos sobreviventes em cada nível da treliça de decodificação é igual a um número $\mathrm{M}$ menor que o número de estados do sistema, possibilitando uma redução de complexidade quando comparado com o Algoritmo de Viterbi.

São apresentadas curvas da probabilidade de erro de bit, $\mathrm{P}_{\mathrm{b}}$, versus $\mathrm{M}$ para vários valores de $E_{b} / N_{0}$. Verificou-se que o desempenho do AM é muito próximo do obtido pelo $\mathrm{AV}$ para valores de $\mathrm{M}$ substancialmente inferiores a $\mathrm{N}_{\sigma}$. Este comportamento é enfatizado quando $\mathrm{E}_{\mathrm{b}} / \mathrm{N}_{0}$ é alto.

São apresentadas curvas de $P_{b}$ versus $E_{b} / N_{0}$ para o $A M$ onde observa-se a degradação de $E_{b} / N_{0}$ para diferentes valores de $M$. Estes dados corroboram o fato que o AlgoritmoMé mais eficiente que o Algoritmo de Viterbi, nos casos investigados.

Este trabalho ilustra, que o aumento do espaço de estados proveniente da precodificação dos símbolos do modulador CPM, pode ser compensado com o uso do Algoritmo-M. A redução de complexidade obtida, possibilita a implementação prática do sistema $\mathrm{CC} / \mathrm{CPM}$.

\section{Referências}

[1] UNGERBOECK, G. Channel Coding with Multilevel Phase Signals. IEEE Trans. Inform. Theory, V. IT-28, p.55-67, Jan. 1982.

Revista da Sociedade Brasileira de Telecomunicações 
2] HO, P.K.; MCLANE, P.J. Spectrum, Distance, and Receiver Complexity of Encoded Continuous Phase Modulation. IEEE trans. Inform. Theory, V. IT-34, p.10211032, Sep. 1988.

3] PIZZI, S.V.; WILSON, S.G. Convolutional Coding Combined with Continuous Phase Modulation. IEEE Trans. on Commun., V. COM-33, p.20-29, Jan. 1985.

4] MULLIGAN, M.G.; WILSON, S.G. An Improved Algorithm for Evaluating Trellis Phases Codes. IEEE Trans. Inform. Theory, V. IT-30, p.846-850, Nov. 1984.

[5] BENEDETTO, S.; BIGLIERI, E.; CASTELLANI, V. Digital Transmission Theory. New Jersey, Prentice-Hall, 1987.

[6] LOO, C. A Statistical Model for a Land Mobile Satellite Link. IEEE Trans. Veh. Technol., V. VT-34, N 3, p.122-127, Aug. 1985.

[7] I.OO, C. et. al. Measurements and Modelling of Land-Mobile Satellite Signal Statistics. 1986 Vehic. Technol. Conf., Dallas, May, 1986.

[8] MCLANE, P.J. et al. PSK and DPSK Trellis Codes for Fast Fading, Shadowed Mobile Satellite Communication Channels. IEEE Trans. on Commun., V.36. $\mathrm{N}^{\circ} 11$, p.1242-1264, 1988.

[9] LOO, C. Measurements and Models of a Mobile-Satellite Link with Application. Proc. GLOBECOM 85, Dec. 1985.

[10] PIMENTEL, C.J.L. Desempenho de Sistemas CPM e de Sistemas CPM Combinados com Códigos Convolucionais. Rio de Janeiro: PUC-RIO, 1990. Tese de Mestrado.

[1i] ANDERSON, J.B.; AUlin, T.; SUNDBERG, C.E. Digital Phase Modulation. New York, Plenum Publishing Company, 1986.

[12] HO, P.; MCLANE. P.J. The Power Spectral Density of Digital Continuous Phase Modulation with Correlated Data Symbols. Part I - The Correlation Function Method. Proc. Inst. Elec. Eng., V.133, Part F, No 1, p.95-105, Feb., 1986.

[13] ANDERSON, J.B. Limited Search Trellis Decoding of Convolutional Codes. IEEE Trans. Inform. Theory, V. IT-35, p.944-955, Sep., 1989.

[14] ANDERSON, J.B.; MOHAN, S. Sequential Coding Algorithms: A Survey and Cost Analysis. IEEE Trans. on Commun., V. COM-32, p. 169-176, Feb., 1984. 
[15] LIN, C.F. A Truncated Viterbi Algorithm Approach to Trellis Codes. Troy, NY. ECSE Department, Rensselaer Polytechnic Institute, 1986. Ph.D. Dissertation.

[16] AULIN, T.A. New Trellis Decoding Algorithm - Analysis and Applications. Gotberg, School of Electrical and Computer Engineering, Chalmers Institute of Tecnology, Sweden, 1985. Technical Report NR2.

\section{A) Resultados da Busca da Distância Euclidiana mínima}

\begin{tabular}{c|cc|c|c}
\hline $\mathrm{h}$ & $\mathrm{d}_{\text {min }}^{2}$ & $\mathrm{~N}_{\min }$ & $\mathrm{h}^{0}$ & $\mathrm{~h}^{1}$ \\
\hline $1 / 2$ & 4.00 & 6 & 1 & 1 \\
$2 / 5$ & 2.65 & 6 & 1 & 1 \\
$1 / 3$ & 3.59 & 8 & 0 & 1 \\
$3 / 10$ & 4.30 & 14 & 1 & 1 \\
$2 / 7$ & 3.90 & 15 & 0 & 1 \\
$1 / 4$ & 4.29 & 10 & 1 & 1 \\
$2 / 9$ & 3.67 & 19 & 1 & 1 \\
$1 / 5$ & 3.19 & 12 & 1 & 1 \\
$1 / 6$ & 2.41 & 12 & 1 & 1 \\
$1 / 7$ & 1.85 & 13 & 1 & 1 \\
$1 / 8$ & 1.45 & 13 & 1 & 1 \\
\hline
\end{tabular}

Tabela 1 - Melhores códigos (4CPM, l-REC, $\mathrm{R}_{\mathrm{c}}=1 / 2, v=2$ ), $\tilde{\mathrm{m}}=1$

\begin{tabular}{c|c|c|c|c}
\hline $\mathrm{h}$ & $\mathrm{d}_{\min }^{2}$ & $\mathrm{~N}_{\min }$ & $\mathrm{h}^{0}$ & $\mathrm{~h}^{1}$ \\
\hline $1 / 2$ & 5,00 & 8 & 0 & 1 \\
$2 / 5$ & 2,65 & 9 & 1 & 2 \\
$1 / 3$ & 4,17 & 11 & 0 & 1 \\
$2 / 7$ & 4,63 & 21 & 1 & 3 \\
$1 / 4$ & 5.24 & 16 & 1 & 2 \\
$2 / 9$ & 4,46 & 21 & 1 & 2 \\
$1 / 5$ & 3,83 & 18 & 1 & 2 \\
$1 / 6$ & 2,88 & 19 & 1 & 2 \\
$1 / 7$ & 2,21 & 19 & 1 & 2 \\
\hline
\end{tabular}

Tabela 2 - Melhores códigos (4CPM, 1-REC, $\left.\mathrm{R}_{\mathrm{c}}=1 / 2, v=3\right), \tilde{\mathrm{m}}=1$ 


\begin{tabular}{c|c|c|c|c}
\hline $\mathrm{h}$ & $\mathrm{d}_{\min }^{2}$ & $\mathrm{~N}_{\min }$ & $\mathrm{h}^{0}$ & $\mathrm{~h}^{1}$ \\
\hline $1 / 2$ & 3,11 & 5 & 1 & 1 \\
$2 / 5$ & 2,10 & 5 & 0 & 1 \\
$1 / 3$ & 3,34 & 7 & 0 & 1 \\
$3 / 10$ & 4,11 & 11 & 0 & 1 \\
$2 / 7$ & 4,45 & 12 & 0 & 1 \\
$1 / 4$ & 5,15 & 12 & 1 & 1 \\
$2 / 9$ & 4,04 & 16 & 1 & 1 \\
$1 / 5$ & 5,53 & 11 & 1 & 1 \\
$1 / 6$ & 3,13 & 14 & 1 & 1 \\
$1 / 7$ & 2,45 & 14 & 1 & 1 \\
$1 / 8$ & 1,96 & 15 & 1 & 1 \\
\hline
\end{tabular}

Tabela 3 - Melhores códigos (4CPM, 1-RC, $\left.R_{c}=1 / 2, v=2\right), \tilde{m}=1$

\begin{tabular}{c|c|c|c|c}
\hline $\mathrm{h}$ & $\mathrm{d}_{\min }^{2}$ & $\mathrm{~N}_{\min }$ & $\mathrm{h}^{0}$ & $\mathrm{~h}^{1}$ \\
\hline $1 / 2$ & 3,68 & 6 & 1 & 1 \\
$2 / 5$ & 3,21 & 8 & 1 & 2 \\
$1 / 3$ & 4,29 & 12 & 0 & 1 \\
$2 / 7$ & 4,45 & 12 & 1 & 3 \\
$1 / 4$ & 5,93 & 17 & 1 & 2 \\
$2 / 9$ & 4,81 & 21 & 1 & 2 \\
$1 / 5$ & 4,39 & 22 & 2 & 1 \\
$1 / 6$ & 3,51 & 20 & 1 & 2 \\
$1 / 7$ & 2,75 & 19 & 1 & 2 \\
\hline
\end{tabular}

Tabela 4 - Melhores códigos (4CPM, 1-RC, $\left.R_{c}=1 / 2, v=3\right), \widetilde{m}=1$

\begin{tabular}{c|c|c|c|c}
\hline $\mathrm{h}$ & $\mathrm{d}_{\min }^{2}$ & $\mathrm{~N}_{\min }$ & $\mathrm{h}^{0}$ & $\mathrm{~h}^{\mathrm{I}}$ \\
\hline $3 / 5$ & 4,10 & 13 & 0 & 1 \\
$1 / 2$ & 4,28 & 11 & 1 & 1 \\
$1 / 3$ & 3,67 & 18 & 0 & 1 \\
$1 / 4$ & 2,26 & 18 & 0 & 1 \\
$2 / 9$ & 1,83 & 19 & 0 & 1 \\
$1 / 6$ & 1,08 & 19 & 0 & 1 \\
$1 / 7$ & 0,80 & 19 & 0 & 1 \\
\hline
\end{tabular}

Tabela 5 - Melhores códigos (4CPM, 2-REC, $\left.R_{c}=1 / 2, v=2\right), \tilde{m}=1$ 


\begin{tabular}{c|c|c|c|c}
\hline $\mathrm{h}$ & $\mathrm{d}_{\text {min }}^{2}$ & $\mathrm{~N}_{\min }$ & $\mathrm{h}^{0}$ & $\mathrm{~h}^{1}$ \\
\hline $3 / 4$ & 3,14 & 6 & 0 & 1 \\
$1 / 2$ & 4,06 & 6 & 1 & 1 \\
$2 / 5$ & 2,80 & 12 & 0 & 1 \\
$1 / 3$ & 3,71 & 9 & 0 & 1 \\
$2 / 7$ & 3,71 & 20 & 0 & 1 \\
$1 / 4$ & 3,72 & 11 & 1 & 1 \\
$2 / 9$ & 3,15 & 19 & 1 & 1 \\
$1 / 5$ & 2,68 & 13 & 1 & 1 \\
$1 / 6$ & 1,98 & 13 & 1 & 1 \\
$1 / 7$ & 1,52 & 13 & 1 & 1 \\
\hline
\end{tabular}

Tabela 6 - Melhores códigos (4CPM, 2-RC, $\left.\mathrm{R}_{\mathrm{c}}=1 / 2, \mathrm{v}=2\right), \widetilde{\mathrm{m}}=1$

\begin{tabular}{c|c|c|c|c}
\hline $\mathrm{h}$ & $\mathrm{d}_{\min }^{2}$ & $\mathrm{~N}_{\min }$ & $\mathrm{h}^{0}$ & $\mathrm{~h}^{1}$ \\
\hline $1 / 2$ & 2,00 & 2 & 0 & 1 \\
$2 / 5$ & 4,24 & 5 & 0 & 1 \\
$1 / 3$ & 3,58 & 3 & 0 & 1 \\
$3 / 10$ & 3,49 & 5 & 0 & 1 \\
$2 / 7$ & 3,57 & 4 & 0 & 1 \\
$1 / 4$ & 2,00 & 2 & 0 & 1 \\
$2 / 9$ & 4,51 & 10 & 0 & 1 \\
$1 / 5$ & 4,55 & 10 & 0 & 1 \\
$1 / 6$ & 4,13 & 11 & 0 & 1 \\
$1 / 7$ & 3,18 & 12 & 1 & 1 \\
\hline
\end{tabular}

Tabela 7 - Melhores códigos ( $\left.8 \mathrm{CPM}, 1-\mathrm{REC}, \mathrm{R}_{\mathrm{c}}=2 / 3, v=2\right), \tilde{\mathrm{m}}=1$

\begin{tabular}{c|c|c|c|c|c}
\hline $\mathrm{h}$ & $\mathrm{d}_{\min }^{2}$ & $\mathrm{~N}_{\min }$ & $\mathrm{h}^{0}$ & $\mathrm{~h}^{\mathrm{l}}$ & $\mathrm{h}^{2}$ \\
\hline $1 / 2$ & 2,01 & 2 & 0 & 1 & 0 \\
$2 / 5$ & 4,80 & 7 & 0 & 0 & 1 \\
$1 / 3$ & 4,85 & 5 & 0 & 0 & 1 \\
$3 / 10$ & 4,21 & 8 & 0 & 0 & 1 \\
$2 / 7$ & 3,82 & 10 & 0 & 0 & 1 \\
$1 / 4$ & 4,00 & 5 & 0 & 0 & 1 \\
$2 / 9$ & 4,51 & 10 & 0 & 1 & 0 \\
$1 / 5$ & 4,41 & 10 & 0 & 1 & 0 \\
$1 / 6$ & 4,09 & 11 & 0 & 1 & 0 \\
$1 / 7$ & 3,18 & 12 & 1 & 1 & 0 \\
\hline
\end{tabular}

Tabela 8 - Melhores códigos ( 8 CPM, 1-REC, $\left.\mathrm{R}_{\mathrm{c}}=1 / 2, \mathrm{v}=2\right), \widetilde{\mathrm{m}}=2$ 


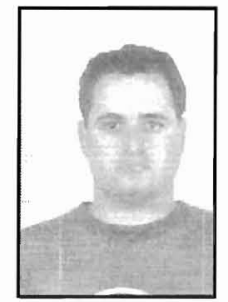

Cecilio Jose Lins Pimentel Nasceu em Recife, Pernambuco, em 7 de junho de 1966. Concluiu o curso de Engenharia Elétrica na Universidade Federal de Pernambuco (UFPe) em 1987 e o Mestrado em Engenharia Elétrica na Pontifícia Universidade Católica do Rio de Janeiro em 1990. No período 1991 a 1992 foi pesquisador associado do Departamento de Informática da UFPe. A partir de janeiro de 1993 é aluno do programa de doutoramento no Departamento de Engenharia Elétrica da Universidade de Waterloo, Canadá. Suas áreas de interesse atual incluem Modulações Codificadas, Transmissão Digital e Comunicações Móveis.

Weiler Alves Finamore nasceu em Cisneiros, Minas Gerais, em 16 de outubro de 1944. Concluiu o curso de Engenharia Elétrica na Pontifícia Universidade Católica do Rio de Janeiro (PUC-Rio) em 1969, o Mestrado e o Doutorado, ambos em Engenharia Elétrica na Universidade de Wisconsin-Madison, Estados Unidos, em 1974 e 1978 respectivamente. Foi professor do Departamento de Engenharia Elétrica da Universidade Federal do Pará no período 1970 a 1979. Foi pesquisador do Centro Científico Rio da IBM-Brasil no período fevereiro de 1990 a setembro de 1991. A partir de julho de 1979 é professor do Departamento de Engenharia Elétrica da PUC-Rio lotado no Centro de Estudos em Telecomunicações da PUC-Rio. Suas áreas de interesse atual incluem Teoria da Informação e Códigos, Processamento Digital de Imagens e Transmissão Digital. 